\title{
Next-to-soft corrections for Drell-Yan and Higgs boson rapidity distributions beyond $\mathrm{N}^{3} \mathrm{LO}$
}

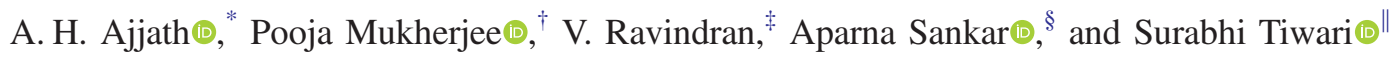 \\ The Institute of Mathematical Sciences, HBNI, Taramani, Chennai 600113, India
}

(Received 8 November 2020; revised 3 February 2021; accepted 28 April 2021; published 8 June 2021)

\begin{abstract}
We present a formalism that resums both soft-virtual (SV) and next-to-SV (NSV) contributions to all orders in perturbative QCD for the rapidity distribution of any colorless particle produced in hadron colliders. Using state-of-the-art results, we determine the complete NSV contributions to third order in the strong coupling constant for the rapidity distributions for Drell-Yan and for Higgs boson in gluon fusion as well as bottom quark annihilation. Using our all-order $z$-space result, we show how the NSV contributions can be resummed in two-dimensional Mellin space.
\end{abstract}

DOI: 10.1103/PhysRevD.103.L111502

\section{INTRODUCTION}

Accurate measurements of observables at the Large Hadron Collider (LHC) and their precise theoretical predictions provide an opportunity to test the Standard Model (SM) with unprecedented accuracy, thereby constraining beyond-the-SM (BSM) scenarios. One of the cleanest observables at the LHC is Drell-Yan (DY) production [1] of on-shell vector bosons $Z$ and $W^{ \pm}$or a pair of leptons, and hence it has received enormous attention from the theory community. Measurements [2-4] of inclusive and differential rates of DY production are used as a standard candle to calibrate the detectors and fit the nonperturbative parton distribution functions (PDFs) [5-9]. Any deviation from the SM predictions can provide crucial information to BSM scenarios, such as $R$-parity-violating supersymmetric models, models with $Z^{\prime}$, and large extra-dimension models $[10,11]$. Similarly, the ongoing measurements of inclusive and differential cross sections [12,13], along with the theoretical predictions [14] on strong and electroweak radiative corrections, help us to probe the symmetrybreaking mechanism and the coupling of the Higgs boson with other SM particles. This is possible owing to the thirdorder QCD predictions for DY production $[15,16]$ and

\footnotetext{
*ajjathah@imsc.res.in poojamukherjee@imsc.res.in

*ravindra@imsc.res.in

aparnas@imsc.res.in

"surabhit@imsc.res.in
}

Published by the American Physical Society under the terms of the Creative Commons Attribution 4.0 International license. Further distribution of this work must maintain attribution to the author(s) and the published article's title, journal citation, and DOI. Funded by SCOAP ${ }^{3}$.
Higgs boson productions in gluon fusion $[14,17,18]$ and bottom-quark annihilation $[19,20]$.

Like inclusive rates, differential ones also get large contributions from logarithms from phase-space boundaries of the final-state particles, thus spoiling the reliability of the fixed-order predictions. These large logarithms can be summed up to all orders in perturbation theory. In the seminal works of Sterman [21] and Catani and Trentadue [22], resummation of leading large logs for the inclusive rates in Mellin space and to differential distribution with respect to $x_{F}$ [22] using double Mellin moments were achieved. Using factorization properties of differential cross sections and renormalization group $(\mathrm{RG})$ invariance, an all-order $z$-space formalism was also developed in Ref. [23] to study the threshold-enhanced contribution to the rapidity distribution of any colorless particle. The formalism was also applied to $Z$ and $W^{ \pm}[24]$ and DY and Higgs production at the $\mathrm{N}^{3} \mathrm{LO}$ level [20,25]. In Ref. [26], the same formalism [23] was used to study the threshold resummation of the rapidity distribution of Higgs bosons and, later, DY production [27]. For different approaches and their applications, see Refs. [28-36].

Besides the threshold logarithms, contributions from subleading logarithms are also present in all of the partonic channels beyond leading order in perturbation theory. These subleading logarithms demonstrate perturbative behavior similar to those from the threshold region, which allows one to study their all-order structure. Such logarithms do appear in inclusive reactions and there has been remarkable progress in understanding them. See Refs. [37-49] for more details. Recently, in a series of articles [50,51], we studied a variety of inclusive reactions to understand these subleading logarithms and found a systematic way to sum them up to all orders in $z$ as well as in Mellin $N$ spaces. The latter provides a resummed prediction in $N$ space for subleading logarithms similar to that of threshold ones. 
The differential distributions often show richer logarithmic structure due to their multidimensional space (spanned by $z_{l}$ or $N_{l}$ ), making it a challenging task to understand the all-order structure. In the present paper, using factorization properties of physical observables and RG invariance, we complete the task of organizing the subleading logarithms in a systematic fashion that is suitable for summing them up to all orders in perturbation theory, in both $z_{l}$ and $N_{l}$ spaces.

\section{THEORETICAL FRAMEWORK}

In the QCD-improved parton model, the rapidity distribution of a colorless state $F$ in hadron-hadron collisions is given by

$$
\begin{aligned}
\frac{d \sigma^{c}}{d y}= & \sigma_{\mathrm{B}}^{c}\left(\tau, q^{2}\right) \sum_{a, b=q, \bar{q}, g} \int_{x_{1}^{0}}^{1} \frac{d z_{1}}{z_{1}} \int_{x_{2}^{0}}^{1} \frac{d z_{2}}{z_{2}} f_{a}\left(\frac{x_{1}^{0}}{z_{1}}, \mu_{F}^{2}\right) \\
& \times f_{b}\left(\frac{x_{2}^{0}}{z_{2}}, \mu_{F}^{2}\right) \Delta_{d, a b}^{c}\left(z_{1}, z_{2}, q^{2}, \mu_{F}^{2}, \mu_{R}^{2}\right),
\end{aligned}
$$

where $\sigma_{B}^{c}\left(\mu_{R}^{2}\right)=\sigma_{B}^{c}\left(x_{1}^{0}, x_{2}^{0}, q^{2}, \mu_{R}^{2}\right)$ is the Born cross section and $\mu_{R}$ is the ultraviolet (UV) renormalization scale. The scaling variables $x_{l}^{0}(l=1,2)$ are defined through the hadronic rapidity $y: y=\frac{1}{2} \ln \left(p_{2} . q / p_{1} . q\right)=\frac{1}{2} \ln \left(x_{1}^{0} / x_{2}^{0}\right)$ and $\tau=q^{2} / S=x_{1}^{0} x_{2}^{0}$. Here $q$ denotes the momentum of the colorless state $F$ and $S=\left(p_{1}+p_{2}\right)^{2}$ is the hadronic centerof-mass energy, with $p_{l}(l=1,2)$ being the momenta of incoming hadrons. For the case of lepton pair production in DY, $\sigma^{c}=d \sigma^{q}\left(\tau, q^{2}, y\right) / d q^{2}$, i.e., its invariant mass distribution, and for the case of Higgs production in gluon fusion or in bottom quark annihilation $\sigma^{c}=\sigma^{g, b}\left(\tau, q^{2}, y\right)$ respectively. The PDFs $f_{c}\left(x_{l}, \mu_{F}^{2}\right)$ of colliding partons $c=$ $q, \bar{q}, g, b$ with momentum fractions $x_{l}(l=1,2)$ are renormalized at the factorization scale $\mu_{F}$. The partonic coefficient functions (CFs) $\Delta_{d, a b}$ are perturbatively calculated in QCD in powers of the strong coupling constant $a_{s}\left(\mu_{R}^{2}\right)=g_{s}^{2}\left(\mu_{R}^{2}\right) / 16 \pi^{2}$ and are functions of the scaling variables $z_{l}=x_{l}^{0} / x_{l}(l=1,2)$. They are obtained from the partonic processes through mass factorization. The UVfinite partonic processes contain soft and collinear divergences associated with the soft gluons and collinear partons, beyond leading order in perturbation theory, which can be removed by summing over degenerate final states and by mass factorization. In this paper we restrict ourselves to partonic CFs of only quark-antiquarkinitiated processes for DY, gluon-gluon, and bottomantibottom-initiated processes for Higgs production. We call them diagonal $\mathrm{CFs}$ (dCFs) $\Delta_{d, a \bar{a}}(a=$ $q, g, b)$. These $\mathrm{dCFs}$ are comprised of contributions from $\delta\left(1-z_{l}\right)$ and $\mathcal{D}_{j}\left(z_{l}\right) \equiv\left(\frac{\ln ^{j}\left(1-z_{l}\right)}{\left(1-z_{l}\right)}\right)_{+}$(namely, SV) and the coefficients regular in $z_{l}$. The leading contributions of the latter near the threshold region $z_{l}=1$ contain terms of the form $\mathcal{D}_{i}\left(z_{l}\right) \ln ^{k}\left(1-z_{j}\right)$ and $\delta\left(1-z_{l}\right) \ln ^{k}\left(1-z_{j}\right)$, with $(l, j=1,2),(i, k=0,1, \ldots)$. We call them next-tosoft-virtual (NSV) contributions. In the Mellin $N_{l}$ space, these terms are of the form $\ln ^{k} N_{j} / N_{l}$, with $(j, l=1,2)$, $(k=0,1 \cdots)$. The dominant SV contribution has been studied in the earlier works of one of the authors in Ref. [23]. In the following, we discuss the NSV contributions of the dCFs in both $z_{l}$ and $N_{l}$ space.

\section{FIXED-ORDER FORMALISM}

Using RG invariance and the factorization properties of differential dCFs [23], the threshold-enhanced SV and NSV terms of dCFs, denoted by $\Delta_{d, c}^{\mathrm{SV}+\mathrm{NSV}}$, are found to exponentiate as

$$
\Delta_{d, c}^{\mathrm{SV}+\mathrm{NSV}}=\left.\mathcal{C} \exp \left(\Psi_{d}^{c}\left(q^{2}, \mu_{R}^{2}, \mu_{F}^{2}, \bar{z}_{1}, \bar{z}_{2}, \epsilon\right)\right)\right|_{\epsilon=0},
$$

where the function $\Psi_{d}^{c}$ is computed in perturbative QCD in $4+\epsilon$ space-time dimensions, and $\bar{z}_{1}=1-z_{1}$ and $\bar{z}_{2}=$ $1-z_{2}$ are the shifted scaling variables. It was shown in Eq. (9) of Ref. [23] that the UV- and IR-finite function $\Psi_{d}^{c}$ can be decomposed in terms of the form factor $F^{c}$, soft distribution $\Phi_{d}^{c}$, and diagonal Altarelli-Parisi (AP) kernels $\Gamma_{c c}$. The soft distribution discussed in Ref. [23], using a $\mathrm{K}+\mathrm{G}$-type Sudakov differential equation, accounts for the soft enhancements associated with the real emissions in the production channel and is universal in nature. This universality ensures that $\Phi_{d}^{c}$ is only sensitive to the initial legs and is blind to the hard process under study. In this paper, we find that the $\mathrm{K}+\mathrm{G}$ equation admits a solution that can account for next-to-soft contributions as well:

$$
\begin{aligned}
\Phi_{d}^{c}= & \sum_{i=1}^{\infty} \hat{a}_{s}^{i}\left(\frac{q^{2} \bar{z}_{1} \bar{z}_{2}}{\mu^{2}}\right)^{i \frac{\epsilon}{2}} S_{\epsilon}^{i}\left[\frac{(i \epsilon)^{2}}{4 \bar{z}_{1} \bar{z}_{2}} \hat{\phi}_{d}^{c,(i)}(\epsilon)\right. \\
& \left.+\frac{i \epsilon}{4 \bar{z}_{1}} \varphi_{d, c}^{(i)}\left(\bar{z}_{2}, \epsilon\right)+\frac{i \epsilon}{4 \bar{z}_{2}} \varphi_{d, c}^{(i)}\left(\bar{z}_{1}, \epsilon\right)\right],
\end{aligned}
$$

where $S_{\epsilon}=\exp \left(\frac{\epsilon}{2}\left[\gamma_{E}-\ln (4 \pi)\right]\right)$, with $\gamma_{E}$ being the EulerMascheroni constant. The first term within the parentheses accounts for the soft contributions, and the remaining two terms correspond to next-to-soft contributions. The soft part of the solution was proposed along with the predictions for Higgs production and DY in Ref. [23] to third order, without $\delta\left(\bar{z}_{1}\right) \delta\left(\bar{z}_{2}\right)$ terms. Later on, Refs. [20,25] gave the complete result for SV. Through mass factorization, the divergent part of the NSV solution cancels against the collinear singularities from AP kernels and the finite part contributes to dCFs. The coefficients $\varphi_{d, c}^{(i)}$ depend on $\bar{z}_{l}$ and $\epsilon$ in such a way that the NSV part is RG invariant provided we sum the series to all orders. In addition, we find that the logarithmic structure of $\Phi_{d}^{c}$ and consequently their predictions remain unaltered under the simultaneous transformation of the exponent in the first 
parentheses and the $z_{l}$ dependence in $\varphi_{d, c}^{(i)}\left(z_{l}, \epsilon\right)$. The AP kernels satisfy

$$
\mu_{F}^{2} \frac{d}{d \mu_{F}^{2}} \mathcal{C} \ln \Gamma_{c c}\left(\mu_{F}^{2}, \bar{z}_{l}\right)=\frac{1}{2} P^{c}\left(a_{s}\left(\mu_{F}^{2}\right), \bar{z}_{l}\right)+\delta P^{c},
$$

where

$P^{c}\left(a_{s}, \bar{z}_{l}\right)=2\left(\frac{A^{c}\left(a_{s}\right)}{\left(\bar{z}_{l}\right)_{+}}+B^{c}\left(a_{s}\right) \delta\left(\bar{z}_{l}\right)+L^{c}\left(a_{s}, \bar{z}_{l}\right)\right)$,

with $A^{c}$ and $B^{c}$ being the cusp and collinear anomalous dimensions, $L^{c}\left(a_{s}, \bar{z}_{l}\right) \equiv C^{c}\left(a_{s}\right) \ln \left(\bar{z}_{l}\right)+D^{c}\left(a_{s}\right)$, and the $\delta P^{c}$ denote NSV and beyond-NSV terms, respectively. We drop $\delta P^{c}$ throughout. The NSV-improved solution $\Phi_{d}^{c}$ results in an integral representation of the finite function $\Psi_{d}^{c}$, which embeds all order information of the massfactorized differential distribution, the mass-factorized differential distribution,

$$
\begin{aligned}
\Psi_{d}^{c}= & \frac{\delta\left(\bar{z}_{1}\right)}{2}\left(\int_{\mu_{F}^{2}}^{q^{2} \bar{z}_{2}} \frac{d \lambda^{2}}{\lambda^{2}} \mathcal{P}^{c}\left(a_{s}\left(\lambda^{2}\right), \bar{z}_{2}\right)+\mathcal{Q}_{d}^{c}\left(a_{s}\left(q_{2}^{2}\right), \bar{z}_{2}\right)\right)_{+} \\
& +\frac{1}{4}\left(\frac { 1 } { \overline { z } _ { 1 } } \left\{\mathcal{P}^{c}\left(a_{s}\left(q_{12}^{2}\right), \bar{z}_{2}\right)+2 L^{c}\left(a_{s}\left(q_{12}^{2}\right), \bar{z}_{2}\right)\right.\right. \\
& \left.\left.+q^{2} \frac{d}{d q^{2}}\left(\mathcal{Q}_{d}^{c}\left(a_{s}\left(q_{12}^{2}\right), \bar{z}_{2}\right)+2 \varphi_{d, c}^{f}\left(a_{s}\left(q_{2}^{2}\right), \bar{z}_{2}\right)\right)\right\}\right)_{+} \\
& +\frac{1}{2} \delta\left(\bar{z}_{1}\right) \delta\left(\bar{z}_{2}\right) \ln \left(g_{d, 0}^{c}\left(a_{s}\left(\mu_{F}^{2}\right)\right)\right)+\bar{z}_{1} \leftrightarrow \bar{z}_{2},
\end{aligned}
$$

where $\quad \mathcal{P}^{c}\left(a_{s}, \bar{z}_{l}\right)=P^{c}\left(a_{s}, \bar{z}_{l}\right)-2 B^{c}\left(a_{s}\right) \delta\left(\bar{z}_{l}\right), \quad q_{l}^{2}=$ $q^{2}\left(1-z_{l}\right)$, and $q_{12}^{2}=q^{2} \bar{z}_{1} \bar{z}_{2}$. The subscript + indicates the standard plus distribution. The function $\mathcal{Q}_{d}^{c}$ in Eq. (6) is given as

$$
\mathcal{Q}_{d}^{c}\left(a_{s}, \bar{z}_{l}\right)=\frac{2}{\bar{z}_{l}} D_{d}^{c}\left(a_{s}\right)+2 \varphi_{d, c}^{f}\left(a_{s}, \bar{z}_{l}\right) .
$$

The splitting function $P^{c}$ and the $\mathrm{SV}$ coefficient $D_{d}^{c}$ are known to third order [26] in QCD. Here $\varphi_{d, c}^{f}$ constitutes the finite part of $\varphi_{d, c}^{(i)}$ in Eq. (3) and is parametrized in the following way:

$$
\begin{aligned}
\varphi_{d, c}^{f}\left(a_{s}\left(\lambda^{2}\right), \bar{z}_{l}\right) & =\sum_{i=1}^{\infty} \sum_{k=0}^{\infty} \hat{a}_{s}^{i}\left(\frac{\lambda^{2}}{\mu^{2}}\right)^{i \frac{\epsilon}{2}} S_{\epsilon}^{i} \varphi_{d, c}^{(i, k)}(\epsilon) \ln ^{k} \bar{z}_{l}, \\
& =\sum_{i=1}^{\infty} \sum_{k=0}^{i} a_{s}^{i}\left(\lambda^{2}\right) \varphi_{d, i}^{c,(k)} \ln ^{k} \bar{z}_{l} .
\end{aligned}
$$

The upper limit on the sum over $k$ is controlled by the dimensionally regularized Feynman integrals that contribute to order $a_{s}^{i}$. The constant $g_{d, 0}^{c}$ in Eq. (6) results from the finite part of the virtual contributions and pure $\delta\left(\bar{z}_{l}\right)$ terms of $\Phi_{d}^{c}$. The exponent $\Psi_{d}^{c}$ that captures both SV and NSV terms to all orders in perturbation theory is one of the main results of this paper.

\section{MATCHING WITH THE INCLUSIVE}

The NSV function $\varphi_{d, c}^{f}$ can be determined at every order in perturbation theory using fixed-order predictions of $\Delta_{d, c}$. Alternatively, we can determine $\varphi_{d, c}^{f}$ from corresponding inclusive cross sections using the relation [23]

$$
\int_{0}^{1} d x_{1}^{0} \int_{0}^{1} d x_{2}^{0}\left(x_{1}^{0} x_{2}^{0}\right)^{N-1} \frac{d \sigma^{c}}{d y}=\int_{0}^{1} d \tau \tau^{N-1} \sigma^{c},
$$

where $\sigma^{c}$ is the inclusive cross section. This relation in the large- $N$ limit gives

$$
\begin{aligned}
& \sum_{i=1}^{\infty} \hat{a}_{s}^{i}\left(\frac{q^{2}}{\mu^{2}}\right)^{\frac{i \epsilon}{2}} S_{\epsilon}^{i}\left[t_{1}^{i}(\epsilon) \hat{\phi}_{d}^{c,(i)}(\epsilon)-t_{2}^{i}(\epsilon) \hat{\phi}^{c,(i)}(\epsilon)\right. \\
& \left.+\sum_{k=0}^{\infty}\left(t_{3}^{(i, k)}(\epsilon) \varphi_{d, c}^{(i, k)}(\epsilon)-t_{4}^{(i, k)}(\epsilon) \varphi_{c}^{(i, k)}(\epsilon)\right)\right]=0
\end{aligned}
$$

Here we keep $\ln ^{k} N$ as well as $\mathcal{O}(1 / N)$ terms for the determination of the SV and NSV coefficients. The constants $\hat{\phi}^{c,(i)}$ and $\varphi_{c}^{(i, k)}$ are the inclusive counterparts to the SV and NSV coefficients, respectively, which are known to third order in QCD for DY $(c=q)$, for Higgs production in gluon fusion $(c=g)$, and in bottom-quark annihilation $(c=b)$ (for NSV, see Ref. [50]). The coefficients are

$$
\begin{aligned}
t_{1}^{i} & =\frac{i \epsilon(2-i \epsilon)}{4 N^{i \epsilon}} \Gamma^{2}\left(1+i \frac{\epsilon}{2}\right), \quad t_{2}^{i}=\frac{i \epsilon(1-i \epsilon)}{2 N^{i \epsilon}} \Gamma(1+i \epsilon), \\
t_{3}^{(i, k)} & =\Gamma\left(1+i \frac{\epsilon}{2}\right) \frac{\partial^{k}}{\partial \alpha^{k}}\left(\frac{\Gamma(1+\alpha)}{N^{\alpha+i \epsilon / 2}}\right)_{\alpha=i \frac{\epsilon}{2}} \\
t_{4}^{(i, k)} & =\frac{\partial^{k}}{\partial \hat{\alpha}^{k}}\left(\frac{\Gamma(1+\hat{\alpha})}{N^{\hat{\alpha}}}\right)_{\hat{\alpha}=i \epsilon} .
\end{aligned}
$$

\section{ALL-ORDER PREDICTION}

In Refs. [20,23,25], we studied the predictive power of the SV part of $\Psi_{d}^{c}$ to dCFs to all orders using lower-order results. Here, in particular, we predict NSV terms of the form $\delta\left(\bar{z}_{l}\right) \ln ^{k} \bar{z}_{j}, n+1 \leq k \leq 2 n-1$, and $\mathcal{D}_{i}\left(z_{l}\right) \ln ^{k} \bar{z}_{j}$ for $i, k=0,1, \ldots, n ; i+k<2 n-1$ at every order $a_{s}^{n}$ provided $\Psi_{d}^{c}$ is known to order $a_{s}^{n-1}$. From $\Psi_{d}^{c}, c=q, b, g$ determined from second-order inclusive results [50], we obtain for the first time the results for the third-order NSV contributions to dCFs, $\Delta_{d, c}$, for both $c=q, b$ and $c=g$ [52]. Further, using the knowledge of third-order results [50] for inclusive reactions and Eq. (10), we have determined the NSV coefficients $\varphi_{d, i}^{c,(k)}$ and dCFs to third order. They will be presented towards the end in a concise form. 


\section{RESUMMATION}

Near the hadronic threshold region, $z_{l} \rightarrow 1$, the PDFs often become large (due to their small momentum fractions) which allows the threshold contributions from CFs to dominate at every order in $a_{s}$. Hence, truncated perturbative predictions become unreliable. In Mellin space, these dominant ones show up as order-one terms of the form $a_{s} \beta_{0} \ln N_{1} N_{2}$ in the large- $N_{l}$ region at every order. Thanks to the all-order integral representation for $\Psi_{d}^{c}$ in Eq. (6) and the RG equation of $a_{s}$, we can resum these terms to all orders. Defining the double Mellin moment of any arbitrary function $F\left(z_{1}, z_{2}\right)$ as $F_{\vec{N}}=\int_{0}^{1} d z_{1} z_{1}^{N_{1}-1} \int_{0}^{1} d z_{2} z_{2}^{N_{2}-1} F\left(z_{1}, z_{2}\right)$, we obtain $\Delta_{d, \vec{N}}^{c}=\tilde{g}_{d, 0}^{c} \exp \left(\Psi_{d, \vec{N}}^{c}\right)$, which can be expanded in terms of $a_{s}: \Delta_{d, \vec{N}}^{c}=\sum_{i=0}^{\infty} a_{s}^{i}\left(\mu_{R}^{2}\right) \Delta_{d, \vec{N}}^{c,(i)}$. The resummed result for $\Psi_{d, \vec{N}}^{c}$ takes the following form:

$$
\begin{aligned}
\Psi_{d, \vec{N}}^{c}= & \left(g_{d, 1}^{c}(\omega)+\frac{1}{N_{1}} \bar{g}_{d, 1}^{c}(\omega)\right) \ln N_{1} \\
& +\sum_{i=0}^{\infty} a_{s}^{i}\left(\frac{1}{2} g_{d, i+2}^{c}(\omega)+\frac{1}{N_{1}} \bar{g}_{d, i+2}^{c}(\omega)\right) \\
& +\frac{1}{N_{1}} \sum_{i=0}^{\infty} a_{s}^{i} h_{d, i}^{c}\left(\omega, N_{1}\right)+\left(N_{1} \leftrightarrow N_{2}\right),
\end{aligned}
$$

where

$$
\begin{aligned}
& h_{d, 0}^{c}\left(\omega, N_{l}\right)=h_{d, 00}^{c}(\omega)+h_{d, 01}^{c}(\omega) \ln N_{l}, \\
& h_{d, i}^{c}\left(\omega, N_{l}\right)=\sum_{k=0}^{i} h_{d, i k}^{c}(\omega) \ln ^{k} N_{l},
\end{aligned}
$$

where $\omega=a_{s} \beta_{0} \ln N_{1} N_{2}$. The SV resummation coefficients, which are comprised of $\tilde{g}_{d, 0}^{c}$ and $g_{d, i}^{c}$, were discussed extensively in Refs. [26,53,54], and so from here onwards we focus on the NSV resummation coefficients, namely, $\bar{g}_{d, i}^{c}$ and $h_{d, i}^{c}$. In $\vec{N}$ space, the use of resummed $a_{s}$ allows us to organize the series in such a way that $\omega$ is treated as order one at every order in $a_{s}$. The coefficient $\bar{g}_{d, 1}^{c}$ is found to be zero.
TABLE I. The all-order predictions for NSV logarithms in $\Delta_{d, \vec{N}}^{c,(i)}$ for a given set of resummation coefficients.

\begin{tabular}{lllll}
\hline \hline Given & & \multicolumn{3}{c}{ Predictions } \\
\cline { 4 - 5 } \cline { 4 - 5 } Resummation coefficients & & $\Delta_{d, \vec{N}}^{c,(2)}$ & $\Delta_{d, \vec{N}}^{c,(3)}$ & $\Delta_{d, \vec{N}}^{c,(i)}$ \\
\hline$\tilde{g}_{d_{0}, 0}^{c}, g_{d, 1}^{c}, g_{d, 2}^{c}, \bar{g}_{d, 1}^{c}, \bar{g}_{d, 2}^{c}, h_{d, 0}^{c}, h_{d, 1}^{c}$ & & $\frac{\ln ^{3} N_{l}}{N_{l}}$ & $\frac{\ln ^{5} N_{l}}{N_{l}}$ & $\frac{\ln ^{(2 i-1)} N_{l}}{N_{l}}$ \\
$\tilde{g}_{d_{0}, 1}^{c}, g_{d, 3}^{c}, \bar{g}_{d, 3}^{c}, h_{d, 2}^{c}$ & & $\frac{\ln ^{4} N_{l}}{N_{l}}$ & $\frac{\ln ^{(2 i-2)} N_{l}}{N_{l}}$ \\
$\tilde{g}_{d_{0}, n-1}^{c}, g_{d, n+1}^{c}, \bar{g}_{d, n+1}^{c}, h_{d, n}^{c}$ & & & $\frac{\ln ^{(2 i-n)} N_{l}}{N_{l}}$ \\
\hline \hline
\end{tabular}

The coefficients $\bar{g}_{d, i+2}^{c}$ are controlled by the universal cusp anomalous dimension $A^{c}$, while the $h_{d, i}^{c}$ 's are controlled by the NSV coefficients $\varphi_{d, c}^{f}$ and $C^{c}, D^{c}$ from $\mathcal{P}^{c}\left(a_{s}, \bar{z}_{l}\right)$. The resummation coefficients $\tilde{g}_{d_{0}, i}^{c}, g_{d, i}^{c}(\omega), \bar{g}_{d, i}^{c}(\omega)$, and $h_{d, i}^{c}(\omega)$ encode the entire all-order information in a systematic fashion through leading, next-to-leading, ..., SV, and NSV logarithms present in the $\Psi_{d}^{c}$. For instance, the knowledge of second-order resummation coefficients, $\left\{\tilde{g}_{d_{0}, 0}^{c}, g_{d, 1}^{c}, g_{d, 2}^{c}, \bar{g}_{d, 1}^{c}, \bar{g}_{d, 2}^{c}, h_{d, 0}^{c}, h_{d, 1}^{c}\right\}$, is sufficient to predict the $\frac{\ln ^{(2 i-1)} N_{l}}{N_{l}}$ of $\Delta_{d, \vec{N}}^{c,(i)}$ for $i>2$ to all orders. We present Table I towards the end, which demonstrates this feature for $\left(\ln ^{k} N_{l} / N_{l}\right)$ terms. In summary, we study the all-order logarithmic structure of the NSV terms in $\vec{N}$ space, and the resummation coefficients till four loops are provided in the Supplementary Material [55].

\section{RESULTS}

We present the third-order NSV results for dCFs, $\Delta_{d, c}$, with $c=q, b$, corresponding to DY processes and for bottom-quark-induced Higgs production after expanding them as $\Delta_{d, c}=\sum_{i=0}^{\infty} a_{s}^{i}\left(\Delta_{d, c}^{\mathrm{SV},(\mathrm{i})}+\Delta_{d, c}^{\mathrm{NSV},(\mathrm{i})}+\cdots\right)$. We have set $\mu_{R}^{2}=\mu_{F}^{2}=q^{2}$ and express the results in terms of $S U\left(N_{c}\right)$ Casimirs, namely, $C_{F}=\left(N_{c}^{2}-1\right) / 2 N_{c}$ and $C_{A}=N_{c}$, and $n_{f}$, the number of active quark flavors:

$$
\begin{aligned}
\Delta_{d, q}^{\mathrm{NSV},(3)}= & C_{F}^{3}\left\{L_{z_{1}}^{5}(-8 \bar{\delta})+L_{z_{1}}^{4}\left(44 \bar{\delta}-40 \overline{\mathcal{D}}_{0}\right)+L_{z_{1}}^{3}\left[\bar{\delta}\left(132+32 \zeta_{2}\right)+160 \overline{\mathcal{D}}_{0}-160 \overline{\mathcal{D}}_{1}\right]\right. \\
& +L_{z_{1}}^{2}\left[-\bar{\delta}\left(\frac{1136}{3}+320 \zeta_{3}+96 \zeta_{2}\right)+\overline{\mathcal{D}}_{0}\left(416+96 \zeta_{2}\right)+416 \overline{\mathcal{D}}_{1}-240 \overline{\mathcal{D}}_{2}\right] \\
& +L_{z_{1}}\left[\bar{\delta}\left(848 \zeta_{3}-\frac{1675}{3}-\frac{88}{3} \zeta_{2}+\frac{192}{5} \zeta_{2}^{2}\right)-\overline{\mathcal{D}}_{0}\left(640+640 \zeta_{3}+192 \zeta_{2}\right)+\overline{\mathcal{D}}_{1}\left(872+192 \zeta_{2}\right)+336 \overline{\mathcal{D}}_{2}-160 \overline{\mathcal{D}}_{3}\right] \\
& +\left[\bar{\delta}\left(\frac{557}{2}-384 \zeta_{5}+496 \zeta_{3}+\frac{700}{3} \zeta_{2}+128 \zeta_{2} \zeta_{3}-\frac{560}{3} \zeta_{2}^{2}\right)-\overline{\mathcal{D}}_{0}\left(697-816 \zeta_{3}-64 \zeta_{2}-\frac{192}{5} \zeta_{2}^{2}\right)\right. \\
& \left.\left.-\overline{\mathcal{D}}_{1}\left(384+640 \zeta_{3}+288 \zeta_{2}\right)+\overline{\mathcal{D}}_{2}\left(456+96 \zeta_{2}\right)+80 \overline{\mathcal{D}}_{3}-40 \overline{\mathcal{D}}_{4}\right]\right\} \\
& +C_{F}^{2} n_{f}\left\{L_{z_{1}}^{4}\left(-\frac{40}{9} \bar{\delta}\right)+L_{z_{1}}^{3}\left(\frac{1040}{27} \bar{\delta}-\frac{160}{9} \overline{\mathcal{D}}_{0}\right)+L_{z_{1}}^{2}\left[\bar{\delta}\left(32 \zeta_{2}-\frac{620}{9}\right)+112 \overline{\mathcal{D}}_{0}-\frac{160}{3} \overline{\mathcal{D}}_{1}\right]\right.
\end{aligned}
$$




$$
\begin{aligned}
& +L_{z_{1}}\left[-\bar{\delta}\left(\frac{9080}{81}+\frac{320}{3} \zeta_{3}+\frac{32}{3} \zeta_{2}\right)-\overline{\mathcal{D}}_{0}\left(\frac{1040}{9}-64 \zeta_{2}\right)+\frac{640}{3} \overline{\mathcal{D}}_{1}-\frac{160}{3} \overline{\mathcal{D}}_{2}\right] \\
& +\left[\bar{\delta}\left(\frac{1999}{27}+\frac{2032}{9} \zeta_{3}-\frac{664}{9} \zeta_{2}+\frac{256}{15} \zeta_{2}^{2}\right)-\overline{\mathcal{D}}_{0}\left(\frac{1448}{9}+\frac{320}{3} \zeta_{3}+\frac{32}{9} \zeta_{2}\right)\right. \\
& \left.\left.+\overline{\mathcal{D}}_{1}\left(64 \zeta_{2}-\frac{200}{3}\right)+96 \overline{\mathcal{D}}_{2}-\frac{160}{9} \overline{\mathcal{D}}_{3}\right]\right\}+C_{A} C_{F}^{2}\left\{L_{z_{1}}^{4}\left(\frac{220}{9} \bar{\delta}\right)+L_{z_{1}}^{3}\left[\bar{\delta}\left(32 \zeta_{2}-\frac{5756}{27}\right)+\frac{880}{9} \overline{\mathcal{D}}_{0}\right]\right. \\
& +L_{z_{1}}^{2}\left[\bar{\delta}\left(\frac{3572}{9}-168 \zeta_{3}-\frac{812}{3} \zeta_{2}\right)+\overline{\mathcal{D}}_{0}\left(96 \zeta_{2}-640\right)+\frac{880}{3} \overline{\mathcal{D}}_{1}\right] \\
& +L_{z_{1}}\left[\bar{\delta}\left(\frac{70763}{81}+424 \zeta_{3}+\frac{20}{3} \zeta_{2}+\frac{48}{5} \zeta_{2}^{2}\right)+\overline{\mathcal{D}}_{0}\left(\frac{6068}{9}-336 \zeta_{3}-512 \zeta_{2}\right)+\overline{\mathcal{D}}_{1}\left(192 \zeta_{2}-\frac{3784}{3}\right)+\frac{880}{3} \overline{\mathcal{D}}_{2}\right] \\
& +\left[\bar{\delta}\left(\frac{2260}{9} \zeta_{2}-\frac{56101}{54}-116 \zeta_{3}+16 \zeta_{2} \zeta_{3}+24 \zeta_{2}^{2}\right)+\overline{\mathcal{D}}_{0}\left(\frac{11351}{9}+\frac{728}{3} \zeta_{3}-\frac{1456}{9} \zeta_{2}+\frac{48}{5} \zeta_{2}^{2}\right)\right. \\
& \left.\left.+\overline{\mathcal{D}}_{1}\left(\frac{1088}{3}-336 \zeta_{3}-448 \zeta_{2}\right)+\overline{\mathcal{D}}_{2}\left(96 \zeta_{2}-592\right)+\frac{880}{9} \overline{\mathcal{D}}_{3}\right]\right\}+C_{A} C_{F} n_{f}\left\{L_{z_{1}}^{3}\left(\frac{176}{27} \bar{\delta}\right)\right. \\
& +L_{z_{1}}^{2}\left[\bar{\delta}\left(\frac{16}{3} \zeta_{2}-\frac{1678}{27}\right)+\frac{176}{9} \overline{\mathcal{D}}_{0}\right]+L_{z_{1}}\left[\bar{\delta}\left(\frac{14648}{81}-\frac{212}{3} \zeta_{2}\right)+\overline{\mathcal{D}}_{0}\left(\frac{32}{3} \zeta_{2}-\frac{3536}{27}\right)+\frac{352}{9} \overline{\mathcal{D}}_{1}\right] \\
& \left.+\left[\bar{\delta}\left(\frac{196}{3} \zeta_{3}-\frac{118984}{729}+\frac{11816}{81} \zeta_{2}-\frac{208}{15} \zeta_{2}^{2}\right)+\overline{\mathcal{D}}_{0}\left(\frac{16952}{81}-\frac{608}{9} \zeta_{2}\right)+\overline{\mathcal{D}}_{1}\left(\frac{32}{3} \zeta_{2}-\frac{3896}{27}\right)+\frac{176}{9} \overline{\mathcal{D}}_{2}\right]\right\} \\
& +C_{F} n_{f}^{2}\left\{L_{z_{1}}^{3}\left(-\frac{16}{27} \bar{\delta}\right)+L_{z_{1}}^{2}\left(\frac{152}{27} \bar{\delta}-\frac{16}{9} \overline{\mathcal{D}}_{0}\right)+L_{z_{1}}\left[\bar{\delta}\left(\frac{32}{9} \zeta_{2}-\frac{1264}{81}\right)+\frac{304}{27} \overline{\mathcal{D}}_{0}-\frac{32}{9} \overline{\mathcal{D}}_{1}\right]\right. \\
& \left.+\left[\bar{\delta}\left(\frac{10856}{729}+\frac{32}{27} \zeta_{3}-\frac{304}{27} \zeta_{2}\right)+\overline{\mathcal{D}}_{0}\left(\frac{32}{9} \zeta_{2}-\frac{1264}{81}\right)+\frac{304}{27} \overline{\mathcal{D}}_{1}-\frac{16}{9} \overline{\mathcal{D}}_{2}\right]\right\} \\
& +C_{A}^{2} C_{F}\left\{L_{z_{1}}^{3}\left(-\frac{484}{27} \bar{\delta}\right)+L_{z_{1}}^{2}\left[\bar{\delta}\left(\frac{4676}{27}-\frac{98}{3} \zeta_{2}\right)-\frac{484}{9} \overline{\mathcal{D}}_{0}\right]+L_{z_{1}}\left[\bar{\delta}\left(\frac{2560}{9} \zeta_{2}-\frac{47386}{81}+200 \zeta_{3}-\frac{176}{5} \zeta_{2}^{2}\right)\right.\right. \\
& \left.+\overline{\mathcal{D}}_{0}\left(\frac{9496}{27}-\frac{176}{3} \zeta_{2}\right)-\frac{968}{9} \overline{\mathcal{D}}_{1}\right]+\left[\bar{\delta}\left(\frac{587684}{729}+192 \zeta_{5}-\frac{21692}{27} \zeta_{3}-\frac{40844}{81} \zeta_{2}+\frac{176}{3} \zeta_{2} \zeta_{3}+\frac{656}{15} \zeta_{2}^{2}\right)\right. \\
& \left.\left.-\overline{\mathcal{D}}_{0}\left(\frac{49582}{81}-176 \zeta_{3}-\frac{856}{3} \zeta_{2}+\frac{176}{5} \zeta_{2}^{2}\right)+\overline{\mathcal{D}}_{1}\left(\frac{11476}{27}-\frac{176}{3} \zeta_{2}\right)-\frac{484}{9} \overline{\mathcal{D}}_{2}\right]\right\}+\left(z_{1} \leftrightarrow z_{2}\right), \\
& \Delta_{d, b}^{\mathrm{NSV},(3)}=\Delta_{d, q}^{\mathrm{NSV},(3)}+\left[C _ { F } ^ { 3 } \left\{L_{z_{1}}^{3}(-96 \bar{\delta})+L_{z_{1}}^{2}\left(288 \bar{\delta}-288 \overline{\mathcal{D}}_{0}\right)+L_{z_{1}}\left[\bar{\delta}\left(471-88 \zeta_{2}\right)+480 \overline{\mathcal{D}}_{0}-576 \overline{\mathcal{D}}_{1}\right]\right.\right. \\
& \left.+\left[-\bar{\delta}\left(\frac{447}{2}+384 \zeta_{3} 148 \zeta_{2}\right)+\overline{\mathcal{D}}_{0}\left(591-88 \zeta_{2}\right)+288 \overline{\mathcal{D}}_{1}-288 \overline{\mathcal{D}}_{2}\right]\right\} \\
& +C_{F}^{2} n_{f}\left\{L_{z_{1}}^{2}(-16 \bar{\delta})+L_{z_{1}}\left[\bar{\delta}\left(\frac{1642}{9}-32 \zeta_{2}\right)-32 \overline{\mathcal{D}}_{0}\right]+\left[-\bar{\delta}\left(\frac{479}{3}-48 \zeta_{2}\right)+\overline{\mathcal{D}}_{0}\left(\frac{1642}{9}-32 \zeta_{2}\right)-32 \overline{\mathcal{D}}_{1}\right]\right\} \\
& +C_{A} C_{F}^{2}\left\{L_{z_{1}}^{2} 88 \bar{\delta}+L_{z_{1}}\left[\bar{\delta}\left(144 \zeta_{3}+256 \zeta_{2}-\frac{9925}{9}\right)+176 \overline{\mathcal{D}}_{0}\right]\right. \\
& \left.+\left[\bar{\delta}\left(\frac{4615}{6}-408 \zeta_{3}-304 \zeta_{2}\right)-\overline{\mathcal{D}}_{0}\left(\frac{10861}{9}-144 \zeta_{3}-256 \zeta_{2}\right)+176 \overline{\mathcal{D}}_{1}\right]\right\} \\
& \left.+C_{A}^{2} C_{F}\left\{L_{z_{1}} 8 \bar{\delta}-[16 \bar{\delta}]\right\}+\left(z_{1} \leftrightarrow z_{2}\right)\right] .
\end{aligned}
$$

Here, $L_{z_{1}}=\ln \left(\bar{z}_{1}\right), \bar{\delta}=\delta\left(\bar{z}_{2}\right), \quad \overline{\mathcal{D}}_{j}=\left(\frac{\ln ^{j}\left(\bar{z}_{2}\right)}{\left(\bar{z}_{2}\right)}\right)_{+}$, and $\zeta_{2}=$ $1.6449 \cdots$ and $\zeta_{3}=1.20205 \cdots$. Complete third-order results for the Higgs production in gluon fusion are already known [52,56]; however, we cannot confirm our results (which were given in Ref. [55]) with them as they are not publicly available. For DY, we have found that our thirdorder prediction is in complete agreement with Ref. [56] for terms of the type $\mathcal{D}_{i}\left(z_{l}\right) \ln ^{j}\left(\bar{z}_{m}\right), \quad i, j \geq 0, l, m=1$, 
2. The remaining $\delta\left(\bar{z}_{l}\right) \ln ^{j}\left(\bar{z}_{m}\right)$ terms in DY and the complete NSV predictions for Higgs production in the bottom-quark annihilation channel at third order are new. Using results up to third order, we can predict the three highest NSV logarithms to all orders. Here, we present the results at fourth order for $\ln ^{j}\left(\bar{z}_{m}\right), j=7,6,5$ :

$$
\begin{aligned}
\Delta_{d, q}^{\mathrm{NSV},(4)}= & C_{F}^{4}\left\{L_{z_{1}}^{7}\left(-\frac{16}{3} \bar{\delta}\right)+L_{z_{1}}^{6}\left(\frac{128}{3} \bar{\delta}-\frac{112}{3} \overline{\mathcal{D}}_{0}\right)+L_{z_{1}}^{5}\left[\bar{\delta}\left(132+96 \zeta_{2}\right)+240 \overline{\mathcal{D}}_{0}-224 \overline{\mathcal{D}}_{1}\right]\right\}+C_{F}^{3} n_{f}\left\{L_{z_{1}}^{6}\left(-\frac{56}{9} \bar{\delta}\right)\right. \\
& \left.+L_{z_{1}}^{5}\left(\frac{1864}{27} \bar{\delta}-\frac{112}{3} \overline{\mathcal{D}}_{0}\right)\right\}+C_{A} C_{F}^{3}\left\{L_{z_{1}}^{6}\left(\frac{308}{9} \bar{\delta}\right)+L_{z_{1}}^{5}\left[\bar{\delta}\left(-\frac{10576}{27}+48 \zeta_{2}\right)+\left(\frac{616}{3} \overline{\mathcal{D}}_{0}\right)\right]\right\} \\
& +C_{F}^{2} n_{f}^{2}\left\{L_{z_{1}}^{5}\left(-\frac{64}{27} \bar{\delta}\right)\right\}+C_{A} C_{F}^{2} n_{f}\left\{L_{z_{1}}^{5}\left(\frac{704}{27} \bar{\delta}\right)\right\}+C_{A}^{2} C_{F}^{2}\left\{L_{z_{1}}^{5}\left(-\frac{1936}{27} \bar{\delta}\right)\right\}+\mathcal{O}\left(L_{z_{1}}^{4}\right)+\left(z_{1} \leftrightarrow z_{2}\right), \\
\Delta_{d, b}^{\mathrm{NSV},(4)}= & \Delta_{d, q}^{\mathrm{NSV},(4)}+\left\{C_{F}^{4}\left[L_{z_{1}}^{5}(-96 \bar{\delta})\right]+\mathcal{O}\left(L_{z_{1}}^{4}\right)+\left(z_{1} \leftrightarrow z_{2}\right)\right\}, \\
\Delta_{d, g}^{\mathrm{NSV},(4)}= & C_{A}^{4}\left\{L_{z_{1}}^{7}\left(-\frac{16}{3} \bar{\delta}\right)+L_{z_{1}}^{6}\left[\frac{692}{9} \bar{\delta}-\frac{112}{3} \overline{\mathcal{D}}_{0}\right]+L_{z_{1}}^{5}\left[\bar{\delta}\left(144 \zeta_{2}-\frac{12224}{27}\right)+\frac{1336}{3} \overline{\mathcal{D}}_{0}-224 \overline{\mathcal{D}}_{1}\right]\right\} \\
& +C_{A}^{3} n_{f}\left\{L_{z_{1}}^{6}\left(-\frac{56}{9} \bar{\delta}\right)+L_{z_{1}}^{5}\left[\frac{796}{9} \bar{\delta}-\frac{112}{3} \overline{\mathcal{D}}_{0}\right]\right\}+C_{A}^{2} n_{f}^{2}\left\{L_{z_{1}}^{5}\left(-\frac{64}{27} \bar{\delta}\right)\right\}+\mathcal{O}\left(L_{z_{1}}^{4}\right)+\left(z_{1} \leftrightarrow z_{2}\right) .
\end{aligned}
$$

This way, we can predict most of the leading NSV terms to all orders in $a_{s}$. In fact, the resummation in $\vec{N}$ space organizes SV and NSV threshold logarithms to all orders, and the resulting resummation coefficients are controlled by anomalous dimensions as well as $\varphi_{d, c}^{f}$ known to a specific order. The knowledge of these coefficients to specific orders in $a_{s}$ is sufficient to predict the infinite tower of SV and NSV logarithms to a specific accuracy. We summarize our findings in Table I. The results for dCFs and the resummation coefficients are provided in the Supplemental Material [55].

\section{SUMMARY}

Using the factorization properties and RG invariance of partonic dCFs we found that, in addition to the SV terms, the NSV contributions also exponentiate for rapidity distributions. The perturbative structure of NSV terms for the differential distribution with respect to rapidity were extensively analyzed for DY and Higgs productions to all orders. Also, the all-order structure is manifested through an integral representation in $z_{l}$ space, which was used to resum the large logarithms in two-dimensional Mellin space in terms of $\omega$. This allowed us to investigate their numerical impact. Our result expressed in twodimensional $z_{l}$ space can be used to obtain leading SV and NSV terms to all orders from the lower-order results as well as from inclusive reactions. We presented the first results for NSV terms of rapidity distributions till thirdorder for DY [56] and Higgs boson production in the bottom-quark annihilation. From the inclusive results known up to third order in $a_{s}$, we also predicted the leading NSV terms to fourth order for the rapidity distributions of DY and Higgs production in both bottomquark annihilation and gluon fusion for the first time. The entire setup advocated in this paper for the study of diagonal partonic channels can be suitably extended to investigate the all-order structure of other potential nondiagonal partonic channels as well.

\section{ACKNOWLEDGMENTS}

We thank J. Michel and F. Tackmann for third-order DY results of rapidity for comparing purposes and C. Duhr and B. Mistlberger for providing third-order results for the inclusive reactions. We also thank P. Mukhopadhyay for useful discussions.
[1] S. Drell and T.-M. Yan, Phys. Rev. Lett. 25, 316 (1970); 25, 902(E) (1970).

[2] T. Affolder et al. (CDF Collaboration), Phys. Rev. D 63, 011101 (2000).

[3] F. Abe et al. (CDF Collaboration), Phys. Rev. Lett. 81, 5754 (1998).

[4] V. Khachatryan et al. (CMS Collaboration), Eur. Phys. J. C 75, 147 (2015).
[5] J. Gao, M. Guzzi, J. Huston, H.-L. Lai, Z. Li, P. Nadolsky, J. Pumplin, D. Stump, and C.-P. Yuan, Phys. Rev. D 89, 033009 (2014).

[6] L. A. Harland-Lang, A. D. Martin, P. Motylinski, and R. S. Thorne, Eur. Phys. J. C 75, 204 (2015).

[7] R. D. Ball et al. (NNPDF Collaboration), J. High Energy Phys. 04 (2015) 040.

[8] J. Butterworth et al., J. Phys. G 43, 023001 (2016). 
[9] S. Alekhin, J. Blumlein, S. Moch, and R. Placakyte, Phys. Rev. D 96, 014011 (2017).

[10] N. Arkani-Hamed, S. Dimopoulos, and G. R. Dvali, Phys. Lett. B 429, 263 (1998).

[11] L. Randall and R. Sundrum, Phys. Rev. Lett. 83, 3370 (1999).

[12] G. Aad et al. (ATLAS Collaboration), Phys. Lett. B 716, 1 (2012).

[13] S. Chatrchyan et al. (CMS Collaboration), Phys. Lett. B 716, 30 (2012).

[14] C. Anastasiou, C. Duhr, F. Dulat, F. Herzog, and B. Mistlberger, Phys. Rev. Lett. 114, 212001 (2015).

[15] C. Duhr, F. Dulat, and B. Mistlberger, Phys. Rev. Lett. 125, 172001 (2020).

[16] T. Ahmed, M. Mahakhud, N. Rana, and V. Ravindran, Phys. Rev. Lett. 113, 112002 (2014).

[17] Y. Li, A. von Manteuffel, R. M. Schabinger, and H. X. Zhu, Phys. Rev. D 90, 053006 (2014).

[18] S. Catani, L. Cieri, D. de Florian, G. Ferrera, and M. Grazzini, Nucl. Phys. B888, 75 (2014).

[19] C. Duhr, F. Dulat, and B. Mistlberger, Phys. Rev. Lett. 125, 051804 (2020).

[20] T. Ahmed, M. K. Mandal, N. Rana, and V. Ravindran, J. High Energy Phys. 02 (2015) 131.

[21] G. F. Sterman, Nucl. Phys. B281, 310 (1987).

[22] S. Catani and L. Trentadue, Nucl. Phys. B327, 323 (1989).

[23] V. Ravindran, J. Smith, and W. L. van Neerven, Nucl. Phys. B767, 100 (2007).

[24] V. Ravindran and J. Smith, Phys. Rev. D 76, 114004 (2007).

[25] T. Ahmed, M. K. Mandal, N. Rana, and V. Ravindran, Phys. Rev. Lett. 113, 212003 (2014).

[26] P. Banerjee, G. Das, P. K. Dhani, and V. Ravindran, Phys. Rev. D 97, 054024 (2018).

[27] P. Banerjee, G. Das, P. K. Dhani, and V. Ravindran, Phys. Rev. D 98, 054018 (2018).

[28] E. Laenen and G. F. Sterman, Resummation for Drell-Yan differential distributions, in 7th Meeting of the Division of Particles Fields of the APS (DPF 92) (NOTE: Dates changed from Oct 13-17), Batavia, IL (1992), https:// inspirehep.net/literature/341157.

[29] G. F. Sterman and W. Vogelsang, J. High Energy Phys. 02 (2001) 016.

[30] A. Mukherjee and W. Vogelsang, Phys. Rev. D 73, 074005 (2006).

[31] P. Bolzoni, Phys. Lett. B 643, 325 (2006).

[32] T. Becher and M. Neubert, Phys. Rev. Lett. 97, 082001 (2006).

[33] T. Becher, M. Neubert, and G. Xu, J. High Energy Phys. 07 (2008) 030.
[34] M. Bonvini, S. Forte, G. Ridolfi, and L. Rottoli, J. High Energy Phys. 01 (2015) 046.

[35] M. A. Ebert, J. K. L. Michel, and F. J. Tackmann, J. High Energy Phys. 05 (2017) 088.

[36] M. Cacciari and S. Catani, Nucl. Phys. B617, 253 (2001).

[37] E. Laenen, L. Magnea, and G. Stavenga, Phys. Lett. B 669, 173 (2008).

[38] E. Laenen, L. Magnea, G. Stavenga, and C. D. White, J. High Energy Phys. 01 (2011) 141.

[39] D. Bonocore, E. Laenen, L. Magnea, L. Vernazza, and C. D. White, Phys. Lett. B 742, 375 (2015).

[40] D. Bonocore, E. Laenen, L. Magnea, S. Melville, L. Vernazza, and C. White, J. High Energy Phys. 06 (2015) 008.

[41] D. Bonocore, E. Laenen, L. Magnea, L. Vernazza, and C. White, J. High Energy Phys. 12 (2016) 121.

[42] V. Del Duca, E. Laenen, L. Magnea, L. Vernazza, and C. White, J. High Energy Phys. 11 (2017) 057.

[43] N. Bahjat-Abbas, D. Bonocore, J. Sinninghe Damsté, E. Laenen, L. Magnea, L. Vernazza, and C. White, J. High Energy Phys. 11 (2019) 002.

[44] G. Soar, S. Moch, J. Vermaseren, and A. Vogt, Nucl. Phys. B832, 152 (2010).

[45] S. Moch and A. Vogt, J. High Energy Phys. 11 (2009) 099.

[46] D. de Florian, J. Mazzitelli, S. Moch, and A. Vogt, J. High Energy Phys. 10 (2014) 176.

[47] M. Beneke, A. Broggio, M. Garny, S. Jaskiewicz, R. Szafron, L. Vernazza, and J. Wang, J. High Energy Phys. 03 (2019) 043.

[48] M. Beneke, M. Garny, S. Jaskiewicz, R. Szafron, L. Vernazza, and J. Wang, J. High Energy Phys. 01 (2020) 094.

[49] M. Beneke, A. Broggio, S. Jaskiewicz, and L. Vernazza, J. High Energy Phys. 07 (2020) 078.

[50] A. Ajjath, P. Mukherjee, and V. Ravindran, arXiv: 2006.06726.

[51] A. Ajjath, P. Mukherjee, V. Ravindran, A. Sankar, and S. Tiwari, J. High Energy Phys. 04 (2021) 131.

[52] F. Dulat, B. Mistlberger, and A. Pelloni, Phys. Rev. D 99, 034004 (2019).

[53] P. Banerjee, P. K. Dhani, M. C. Kumar, P. Mathews, and V. Ravindran, Phys. Rev. D 97, 094028 (2018).

[54] A. A. H. T. Ahmed, P. Mukherjee, V. Ravindran, and A. Sankar, arXiv:2010.02980.

[55] See Supplemental Material at http://link.aps.org/ supplemental/10.1103/PhysRevD.103.L111502 for all of our results in Mathematica format.

[56] G. Lustermans, J. K. L. Michel, and F. J. Tackmann, arXiv: 1908.00985 . 